\title{
A First Case of Acute Cerebellitis Associated with Coronavirus Disease (COVID-19): a Case Report and Literature Review
}

\author{
Nima Fadakar ${ }^{1}$ - Sara Ghaemmaghami ${ }^{1}$. Seyed Masoom Masoompour ${ }^{2} \cdot$ Babak Shirazi Yeganeh $^{3} \cdot$ Ali Akbari $^{4}$. \\ Sedighe Hooshmandi ${ }^{5} \cdot$ Vahid Reza Ostovan $^{1}$ (D)
}

Published online: 31 July 2020

(C) Springer Science+Business Media, LLC, part of Springer Nature 2020

\begin{abstract}
Novel coronavirus (severe acute respiratory syndrome-coronavirus-2: SARS-CoV-2), which originated from Wuhan, China, has spread to the other countries in a short period of time. We report a 47-year-old male who was admitted to our hospital due to suffering from progressive vertigo and ataxia for 7 days prior to the admission. Neurological examination revealed cerebellar dysfunction, and brain magnetic resonance imaging (MRI) depicted edema of the cerebellar hemisphere associated with leptomeningeal enhancement. Cerebrospinal fluid (CSF) analysis showed mild lymphocytic pleocytosis, elevated protein, and lactate dehydrogenase. SARS-CoV-2 RNA was detected in the oropharyngeal/nasopharyngeal and CSF specimens. As a result, treatment with lopinavir/ritonavir was initiated, and patient symptoms and signs improved significantly during the course of hospitalization. To the best of our knowledge, this is the first case of acute cerebellitis associated with COVID-19 disease which is reported in the literature so far.
\end{abstract}

Keywords COVID-19 $\cdot$ SARS-COV-2 $\cdot$ Ataxia $\cdot$ Vertigo $\cdot$ Cerebellitis $\cdot$ Magnetic resonance imaging

\section{Introduction}

In December 2019, novel coronavirus (severe acute respiratory syndrome-coronavirus-2: SARS-CoV-2) arose from the city of Wuhan, Hubei Province, China. The disease has rapidly expanded into the other countries and developed into a pandemic [1]. Angiotensin-converting enzyme 2(ACE-2) and dipeptidyl peptidase 4 (DPP4) receptors are rarely expressed in the central nervous system except in certain circumstances, such as temporal lobe epilepsy and neurodegenerative

Vahid Reza Ostovan

ostovanv@gmail.com

1 Clinical Neurology Research Center, Shiraz University of Medical Sciences, Shiraz 7193635899, Iran

2 Non-Communicable Diseases Research Center, Shiraz University of Medical Sciences, Shiraz, Iran

3 Pathology Department, Shiraz University of Medical Sciences, Shiraz, Iran

4 Anesthesiology Department, Shiraz University of Medical Sciences, Shiraz, Iran

5 Medical Imaging Research Center, Shiraz University of Medical Sciences, Shiraz, Iran disorders $[2,3]$. Several preliminary reports demonstrated neurological manifestations in the patients infected with SARS-CoV-2 [4, 5]. Central nervous system involvement usually occurs in severe cases of SARS-CoV-2 infection; however, according to the recent reports, neurological manifestations may be the presenting features of COVID-19 [6, 7]. Herein, we report the first case of acute cerebellitis in association with COVID-19 that is confirmed by the detection of SARS-CoV-2 RNA in the cerebrospinal fluid (CSF).

\section{Case Report}

A 47-year-old man presented to our emergency department complaining of body pain, progressive vertigo, headache, and ataxia. He had a history of fatigue, generalized body pain, and an occasional cough for 10 days before admission. After 3 days, progressive vertigo, headache, and ataxia were added to his previous symptoms. Vertigo was not accompanied by nausea, vomiting, tinnitus, or hearing loss. He had no history of change or loss of taste and smell. Ataxia and vertigo were of modest severity, yet they interfered with the patient's daily activities. He had not met any physician during these 10 days and had only used OTC drugs. His past medical history 
$(\mathrm{PMH})$ was unremarkable. On arrival at the hospital, he was awake and oriented, and his vital signs were as follows: temperature $38{ }^{\circ} \mathrm{C}$, blood pressure $90 / 60 \mathrm{mmHg}$, pulse rate 94 beats/min, and respiratory rate 16 breaths/min with oxygen saturation $(\mathrm{SpO} 2)$ of $95 \%$ on room air. Neurological examination revealed an ataxic gait and mild dysarthria. Cerebellar examination was noticeable for impaired tandem gait, widebased and ataxic gait, head titubation, mild truncal swaying while sitting on a bed, irregular rapid alternating hand movements, mild dysarthria, and dysmetria in both upper and lower extremities in finger to nose and heel to shin, respectively. Furthermore, hypermetric vertical and horizontal saccades with normal velocity, instability of visual fixation in the primary position, saccadic pursuit (saccade superimposed on a pursuit eye movement), loss of optokinetic nystagmus, impaired vestibular suppression response, and end gaze rotational nystagmus were noted in examination of eye movements. On admission, the patient scored 14 out of 40 according to the scale for the assessment and rating of ataxia (SARA) scoring [8]. Otherwise, the neurological examination was unremarkable. Initial lab tests showed a normal count in the following factors: white cell $\left(8.7 \times 10^{3} /\right.$ ul with lymphocyte count $32 \%$, normal: $\left.4.8-10.8 \times 10^{3} / \mathrm{ul}\right)$, C-reactive protein $(2 \mathrm{mg} / \mathrm{l}$, normal:0-6 mg/l), lactate dehydrogenase (267 u/1, normal: 200 $480 \mathrm{u} / \mathrm{l})$, serum iron (97 $\mu \mathrm{g} / \mathrm{l}$, normal: 40-168 $\mu \mathrm{g} / \mathrm{l})$, D-dimer (160 ng/ml FEU, normal: up to $880 \mathrm{ng} / \mathrm{ml} \mathrm{FEU),} \mathrm{liver} \mathrm{func-}$ tion test (LFT), thyroid function test (TFT), thyroid peroxidase antibody (anti-TPO) and antigliadin IgA and IgG antibodies, and elevated ferritin level $(879 \mathrm{ng} / \mathrm{ml}$, normal: 18 $270 \mathrm{ng} / \mathrm{ml}$ ). Viral markers including human immunodeficiency virus (HIV), the hepatitis $\mathrm{C}$ virus (HCV) antibody, and the hepatitis $\mathrm{B}$ surface antigen ( $\mathrm{HBs} \mathrm{Ag}$ ) were negative. Brain magnetic resonance imaging (MRI) displayed bilateral cerebellar hemispheres as well as vermis hyperintensities and edema in fluid-attenuated inversion recovery (FLAIR) with cortical-meningeal enhancement of cerebellum in T1weighted with gadolinium sequences (Fig. 1). Due to a previous history of flu-like symptoms, spiral chest CT-scan and real-time reverse-transcriptase polymerase chain reaction (RT-PCR) for SARS-CoV-2 were performed using oropharyngeal/nasopharyngeal and cerebrospinal fluid (CSF) specimens. Spiral chest CT-scan proved insignificant and showed no evidence of pneumonia or its related changes. CSF opening pressure was $250 \mathrm{~mm}$ of $\mathrm{H} 2 \mathrm{O}$, and analysis demonstrated mild pleocytosis, normal glucose, elevated protein, and lactate dehydrogenase $\left(10 / \mathrm{mm}^{3}\right.$ with $80 \%$ lymphocyte, $60 \mathrm{mg} / \mathrm{dl}, 58 \mathrm{mg} / \mathrm{dl}$, and $134 \mathrm{u} / \mathrm{l}$, respectively), as well as negative gram stain, culture, and cytology. Polymerase chain reaction (PCR) tests for herpes simplex virus, varicella zoster, Epstein-Barr virus, influenza virus, and $\mathrm{IgM} / \mathrm{IgG}$ for Borrelia were negative in the CSF. Moreover, paraneoplastic and autoimmune antibody tests including anti-Yo, anti-Hu, anti-Ri, anti-Tr(or anti-delta/notch-like epidermal growth factorrelated receptor [DNER]), anti-Purkinje cell cytoplasmic antibody 2 (PCA 2), anti-glutamic acid decarboxylase (GAD), anti-zinc-finger protein 4 (Zic 4), anti-glial nuclear antibody(AGNA), anti-sox1, anti-metabotropic glutamate receptor 1 (mGluR1), anti-CV2, anti-paraneoplastic antigen $\mathrm{Ma}$ 2(PNMA2), anti-N-methyl-D- aspartate (NMDA) receptor, anti-contactin-associated protein-2 (CASPR2), and leucinerich glioma-inactivated protein 1 (LGI 1) performed on the $\mathrm{CSF}$ and serum were negative. CSF proved positive for the SARS-CoV-2 viral nucleic acid. Also, SARS-CoV-2 RNA was detected in the oropharyngeal and nasopharyngeal specimens. He did not develop dyspnea, difficulty in breathing, chest pain, or hypoxemia during the course of hospitalization. Due to positive SARS-CoV-2 PCR in the CSF and also the clinico-radiological evidence of acute cerebellitis, the patient was immediately treated with lopinavir/ritonavir administered 400/100 mg twice daily for 14 days. During the course of his hospitalization, neither steroids nor IVIg was prescribed owing to the alleviation of his symptoms by antiviral therapy. Although CSF serial sampling was not performed because the patient did not give his consent, PCR for SARS-CoV-2 was rechecked from oropharyngeal and nasopharyngeal specimens 10 days after the initiation of treatment which became
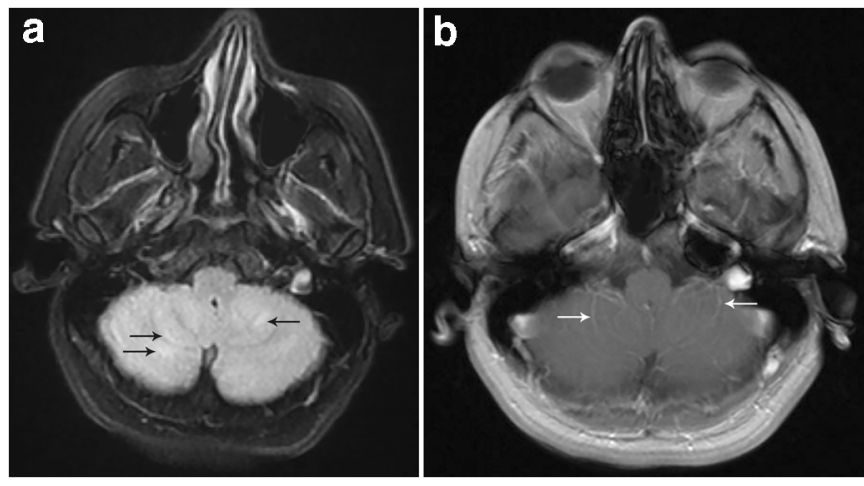

Fig. 1 a, c Fluid-attenuated inversion recovery (FLAIR) MR images showed hyperintensities and edema of the bilateral cerebellar hemispheres as well as vermis (black arrows). b, d Axial contrast-
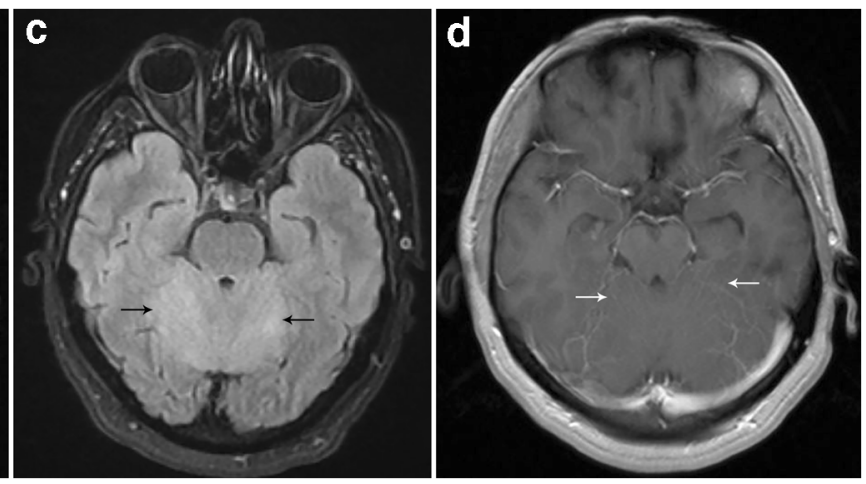

enhanced T1-weighted MR images showed cerebellar corticalmeningeal enhancement, corresponding to the region of cerebellar swelling (white arrows) 
undetectable. At the end of a 14-day course of treatment, the patient showed a marked amelioration of vertigo, and his SARA score decreased 5 points, reaching 9 out of 40 . After 1 month, his ataxia had also improved significantly, and his SARA score dropped to 3 out of 40 . Written informed consent was obtained from the patient for the publication of his anonymous information in this case report.

\section{Specimen Collection and Diagnostic Testing}

Oropharyngeal/nasopharyngeal specimens were collected via synthetic fiber swabs and placed into a single sterile tube containing 2-3 ml virus transfer media (VTM). Two milliliters of CSF was poured into another sterile tube comprising VTM. At the molecular diagnostic laboratory, as a first step, RNA was purified from the clinical samples using the MagNA Pure 96 system (Roche, Penzberg, Germany). Then envelope protein (E) gene screening for SARS-related coronavirus was performed using a one-step master mix kit, and an increase of more than 35 in the cycle threshold (CT) was deemed as a positive result. Afterwards, a confirmatory test with RNAdependent RNA polymerase (RdRp) gene was conducted. Additionally, to determine the quality of the PCR run, human specimen controls including negative (extraction) and internal controls were applied using RNase $\mathrm{P}$ gene.

\section{Discussion}

Acute cerebellitis or acute cerebellar ataxia is an uncommon inflammatory process manifested by cerebellar swelling and dysfunction [9]. Although cerebellitis usually happens as a postinfectious disorder and is more common in children, parainfectious etiopathogenesis and occurrence in adulthood have been well reported in the literature $[9,10]$. Concerning the previously reported neurological manifestations of COVID-19, this is the first case of SARS-CoV-2 associated with acute cerebellitis. In 2005, Gu et al. [11] examined the autopsies of individuals infected with severe acute respiratory syndrome (SARS) virus, which showed the presence of SARS-COV viral particles and genomic sequences in the nervous system. Lu et al. [12] concluded that SARS-CoV-2 had a genomic similarity of about $79 \%$ and a homogenous receptorbinding structure to that of SARS-COV. Therefore, SARSCoV-2 might exhibit neurotropic characteristics like the SARS-COV virus.

Coronavirus can damage the nervous system through several mechanisms including direct infection, hypoxia, ACE-2, and immune-mediated [13]. Taking into account the clinical course of symptoms, presence of fever, increased intracranial pressure, and extraction of SARS-CoV-2 RNA from CSF specimen, direct viral invasion of the nervous system is the more probable pathogenic mechanism in our patient [14]; however, the postinfectious immune-mediated reaction is the most common pathogenesis presumed for acute cerebellar ataxia and absence of extracerebellar symptoms such as altered mental status, cerebral focal sign, and seizure in our patient might implicate the diagnosis of autoimmunemediated cerebellar ataxia $[9,14]$. Lack of consecutive CSF sampling during the course of the therapy and evaluating the correlation between response to the treatment and the disappearance of SARS-COV-2 RNA from CSF constitute the limitations of our report. Hence, more cases with precise reports of virus PCR in the CSF during the course of the disease would be required to more precisely determine and explain which of the two mechanisms (direct nervous system invasion or delayed autoimmunity) are contributing more to the pathophysiology of acute cerebellitis associated with COVID-19. Dizziness has been described in the prior case series with a frequency of $16 \%$ among SARS-COV-2-infected individuals $[5,15]$. However, vertigo has not been reported in the literature so far. Therefore, our findings expand the spectrum of the neurological diseases associated with SARS-CoV-2. As we are living in the era of COVID-19 pandemic, the prompt diagnosis of new cases might be considered the main step in controlling and terminating the spread of this infectious disease. Therefore, neurologists should be aware of different neurological presentations of COVID-19 and should not underestimate any neurological findings.

\section{Conclusions}

A SARS-CoV-2 infection should be considered in the case of any patient who develops clinical features consistent with acute cerebellitis, and patients must be promptly diagnosed and quarantined in order to stop the propagation of the disease.

\section{Compliance with Ethical Standards}

All of the actions described in this study have been conducted in accordance with ethical guidelines. Informed consent was obtained from the patient for the publication of his anonymous information in this manuscript.

Conflict of Interest The authors declare that they have no conflict of interests.

\section{References}

1. Wang D, Hu B, Hu C, Zhu F, Liu X, Zhang J, et al. Clinical characteristics of 138 hospitalized patients with 2019 novel coronavirus-infected pneumonia in Wuhan, China. JAMA. 2020;323(11):1061-9.

2. Argañaraz GA, Konno AC, Perosa SR, Santiago JFC, Boim MA, Vidotti DB, et al. The renin-angiotensin system is upregulated in the cortex and hippocampus of patients with temporal lobe epilepsy 
related to mesial temporal sclerosis. Epilepsia. 2008;49(8):134857.

3. Ge J, Barnes NM. Alterations in angiotensin AT1 and AT2 receptor subtype levels in brain regions from patients with neurodegenerative disorders. Eur J Pharmacol. 1996;297(3):299-306.

4. Helms J, Kremer S, Merdji H, Clere-Jehl R, Schenck M, Kummerlen C, et al. Neurologic features in severe SARS-CoV-2 infection. N Engl J Med. 2020. https://doi.org/10.1056/ NEJMc2008597.

5. Mao L, Jin H, Wang M, Hu Y, Chen S, He Q, et al. Neurological manifestations of hospitalized patients with coronavirus disease 2019 in Wuhan, China. JAMA Neurol. 2020;77:683. https://doi. org/10.1001/jamaneurol.2020.1127.

6. Moriguchi T, Harii N, Goto J, Harada D, Sugawara H, Takamino J. A first case of meningitis/encephalitis associated with SARSCoronavirus-2. Int J Infect Dis. 2020;94:55-8.

7. Zhao H, Shen D, Zhou H, Liu J, Chen S. Guillain-Barré syndrome associated with SARS-CoV2 infection: causality or coincidence? Lancet Neurol. 2020;19(5):383-4.

8. Schmitz-Hübsch T, Tezenas du Montcel S, Baliko L, Berciano J, Boesch S, Depondt C, et al. Scale for the assessment and rating of ataxia. Development of a new clinical scale. Neurology. 2006;66(11):1717-20.

9. Sawaishi Y, Takada G. Acute cerebellitis. Cerebellum. 2002;1(3): 223-8.
10. MM, Najem CE. Cerebellitis associated with influenza a (H1N1)pdm09, United States, 2013. Emerg Infect Dis. 2014;20(9):1578-80.

11. Gu J, Gong E, Zhang B, Zheng J, Gao Z, Zhong Y. Multiple organ infection and the pathogenesis of SARS. J Exp Med. 2005;202(3): 415-24.

12. Lu R, Zhao X, Li J, Niu P, Yang B, Wu H, et al. Genomic characterisation and epidemiology of 2019 novel coronavirus: implications for virus origins and receptor binding. Lancet. 2020;395(10224):565-74.

13. Wu Y, Xu X, Chen Z, Duan J, Hashimoto K, Yang L, et al. Nervous system involvement after infection with COVID-19 and other coronaviruses. Brain Behav Immun. 2020;87:18-22. https://doi. org/10.1016/j.bbi.2020.03.031.

14. Sivaswamy L. Approach to acute ataxia in childhood: diagnosis and evaluation. Pediatr Ann. 2014;43(4):153-9.

15. Song F, Shi N, Shan F, Zhang Z, Shen J, Lu H, et al. Emerging 2019 novel coronavirus (2019-nCoV) pneumonia. Radiology. 2020;295(1):210-7.

Publisher's Note Springer Nature remains neutral with regard to jurisdictional claims in published maps and institutional affiliations. 